\title{
OBSERVER BASED OPTIMAL CONTROL OF MR DAMPERS
}

\author{
Mehmet A. Eroglu ${ }^{1}$, Neil D. Sims ${ }^{1}$ \\ ${ }^{1}$ Department of Mechanical Engineering, The University of Sheffield, U.K. \\ (M.eroglu@ sheffield.ac.uk)
}

\begin{abstract}
Magneto-rheological, or MR, dampers are one of the most promising semi-active control devices for protecting civil engineering structures, vehicles, ships, or aircraft from the damaging effects of dynamic loading. They have many advantages over alternative technologies, such as low power requirement, reliability, and low cost. A wide range of control schemes have been considered for MR dampers, with no general consensus on the most appropriate approach. Research at the University of Sheffield has focused on feedback linearization, but this requires measurement of the damping force which increases the complexity of the system. This study aims to overcome this problem and improve the vibration absorbability of the system by investigating the application of observer based optimal control to the force-feedback linearization of an MR damper. The proposed force-feedback linearization chose the set point force as proportional to the piston velocity. But in this study, in order improve the performance of the system, the desired set point force is chosen to be the optimal control force. The implementation of the optimal control theory requires the measurement of the system states (displacement and velocity of the mass), are provided by the observer as well. Due to passivity limitation of the MR damper the set point force is diverted to the zero at the active region to satisfy the passivity theory of Karnopp.

The results of this study is compared to observer based force-feedback linearization algorithm and it is concluded that the proposed control system is able to reduce the displacement transmissibility of the damped system better then the compared one.
\end{abstract}

Keywords: Smart Fluids, Optimal Control, Observer, Force-feedback linearization

\section{INTRODUCTION}

Scientists have been studying the vibration theory since the first quarter of the twentieth century [5-17]. Some of the earliest research was done by Timoshenko [17] and Den Hartog [5] who described the primary solutions to vibration problem of the engineering structures. These solutions were achieved by applying passive devices to an engineering system such as a structure, turbine, vehicle, or bridges. The passive suspension system has three elements which are isolated masses, the linear spring elements and the passive viscous damper that does not require any power sources [4]. Depending on the relative velocity of the damper, it dissipates energy which is not controllable as the suspension properties remain fixed. This makes the performance of the passive systems highly dependent on the excitation bandwidth. For the large excitation 
bandwidth the performance will decrease. Due to this passive systems are optimal for specific conditions [12].

This major downside of the passive devices was soon recognized as a problem [5]. In order to overcome this limitation scientists developed the active and semi-active systems, which are able to change their absorbing behaviour according to measured data [5].

In the 1950's and 60's, active suspension systems started to get considerable attention in order to avoid this limitations of the passive systems for vibration control [3]. Energy dissipation and storage from and to the vibrating system is achieved by a hydraulic actuator. Here, electrohydraulic servo-valves control the flow rate of high-pressure fluid that is pumped into and out of the actuator. As a result, better performance can be achieved over wide ranging excitation conditions by using appropriate sensors and control logic.

In the present day one of the most promising suspension systems is the semi-active system which deals with smart fluids. The flow resistance of these fluids changes very quickly and continuously by applying an electric or magnetic field [3].

The performance of semi-active systems almost reaches that of active systems while keeping the weight, cost and complexity of the system similar to passive systems [7, 12]. Semiactive systems provide a means to control energy storage and/or dissipation. But such systems cannot increase the energy of the system like active systems, so the power requirement of these systems is very low.

Magneto-rheological, or MR, dampers are one of the most promising semi-active control devices for protecting civil engineering structures, vehicles, ships, or aircraft from the damaging effects of dynamic loading. They have many advantages over alternative technologies, such as low power requirement, reliability, and low cost. A wide range of control schemes have been considered for MR dampers, with no general consensus on the most appropriate approach. Research at the University of Sheffield has focused on feedback linearization [9], but this requires measurement of the damping force which increases the complexity of the system.

The present study aims to overcome this problem by investigating the application of observer based control to the optimal feedback linearization of an MR damper. A single-degreeof freedom structure is chosen as the basis for study, as shown schematically in Figure 1. With reference to Figure 1, the aim is to perform optimal force-feedback linearization of the MR damper (so that it can perform as an arbitrary semi-active force generator) using an observation of the feedback force, rather than a measured value. The present report considers a simplified modelling approach to this problem, although corresponding experiments are planned for the near future. 


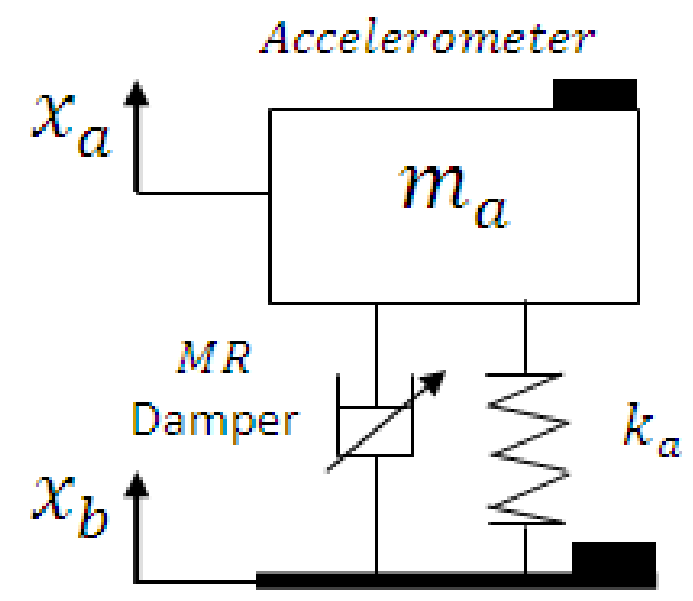

Figure 1: Mass-spring-damper system.

\section{MODELLING OF SDOF SYSTEM}

Figure 1 represents the single degree of freedom (SDOF) mass isolator system, where the $m_{a}$ represents the mass of structure, $k_{a}$ represents the spring stiffness and MR damper represents the controllable damper. This system has two states which may be conveniently chosen as, absolute displacement of the structural mass $x_{a}$, and the absolute velocity of the structural mass $\dot{x}_{a}$. Between the structural mass and the base the controllable MR damper exerts a force $f_{m r}$. The controllable damper accepts a control signal current $I$ which is used to generate the damper force $f_{m r}$.

Using these two states, the system of Figure 1 can be written in state space form as

$$
\begin{aligned}
& \dot{x}(t)=\mathrm{A} x(t)+\mathrm{B} f_{m r}(t)+\mathrm{E} d(t) . \\
& y(t)=\mathrm{C} x(t)+\mathrm{D} f_{m r}(t)+\mathrm{G} d(t) .
\end{aligned}
$$

Here the measured output $y$ is the structural acceleration $y=\ddot{x}_{a}$. The disturbance is the ground excitation, $d=x_{b}$ and the state vector $x$ is defined as

$$
x=\left[\begin{array}{l}
x_{a} \\
\dot{x}_{a}
\end{array}\right]
$$

The matrices $A, B, C, D, E$ and $G$ are then given by:

$$
\mathrm{A}=\left[\begin{array}{cc}
0 & 1 \\
\frac{-k_{a}}{m_{a}} & 0
\end{array}\right], \mathrm{B}=\left[\begin{array}{c}
0 \\
\frac{-1}{m_{a}}
\end{array}\right], \mathrm{E}=\left[\begin{array}{c}
0 \\
\frac{k_{a}}{m_{a}}
\end{array}\right], \mathrm{C}=\left[\begin{array}{ll}
\frac{-k_{a}}{m_{a}} & 0
\end{array}\right], \mathrm{D}=\left[\frac{-1}{m_{a}}\right], \mathrm{G}=\left[\frac{k_{a}}{m_{a}}\right]
$$


In order to model the nonlinear behaviour of the MR damper, the lumped-parameter model shown schematically in Figure 2 was used [8].

(a)
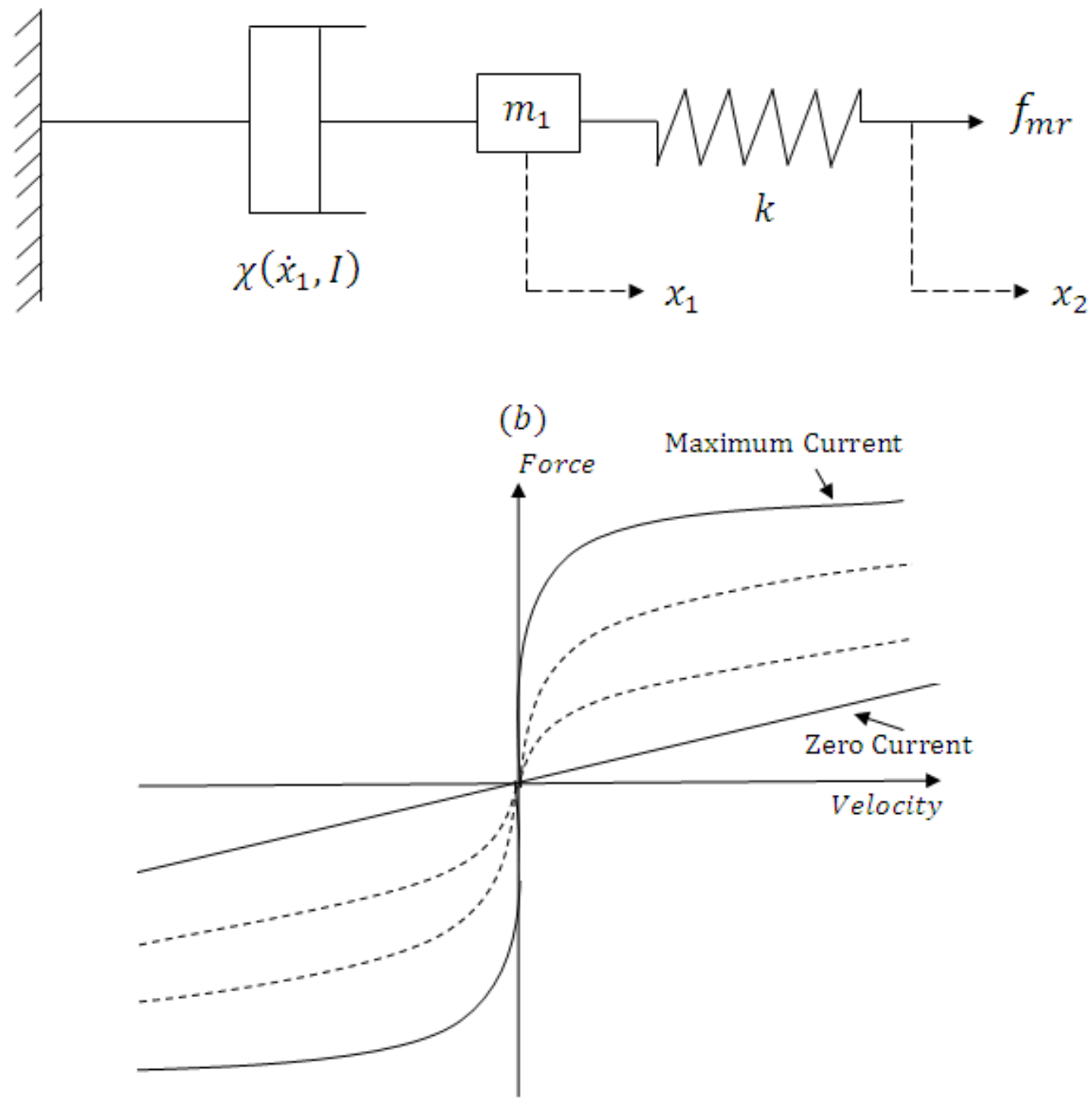

Figure 2: (a) The lumped-parameter model of MR damper. (b) Schematic representation of quasisteady damper function $\chi$.

Figure 2 shows the lumped-parameter model and the force/velocity characteristic of the MR damper. Here, the stiffness element $k$ represents the compressibility of the MR fluid, whilst 
the mass $m_{1}$ represents its inertia. The nonlinear damper represents the Bingham plastic nature of the MR fluid flow. In the absence of an applied current the force/velocity characteristics is a straight line through the origin, indicating Newtonian behaviour as seen in Figure 2(b). As the magnetic field increased up to a maximum current so the yield force produced by the damper is seen to increase. In Figure 2(a) the valve flow which is quasi-steady is described by the nonlinear function $\chi$ and is a function of the quasi steady velocity of $\dot{\boldsymbol{x}}_{\mathbf{1}}$ and the control signal $I$ to the smart damper:

$$
\chi\left(\dot{x}_{1}, I\right)=\left\{\begin{array}{cc}
C_{\text {pre }} \dot{x}_{1} & \dot{x}_{1} \leq F_{y} / C_{\text {pre }} \\
C_{\text {post }} \dot{x}_{1}+F_{y} \operatorname{sgn}\left(\dot{x}_{1}\right) & \dot{x}_{1}>F_{y} / C_{\text {pre }}
\end{array}\right\} .
$$

Where $C_{\text {pre }}, C_{\text {post }}$ and $F_{y}$ are the modal parameters referred to the Sims et al. work [8]. The force produced by the controllable MR damper is:

$$
f_{m r}=k\left(x_{2}-x_{1}\right)
$$

Here the $x_{1}$ represents the absolute displacement of the fluid inertia and $x_{2}$ represents the relative displacement of the piston.

\section{OBSERVER DESIGN}

The aim of the observer is to estimate the states, piston velocity and damper force of the SDOF mass isolator problem for the implementation of the optimal force feedback linearization. There could be other ways to measure the damper force, piston velocity and the system states such as using a load cell (force measurement), and LVDT sensors (displacement measurement). However, the implementation of the load cell and LVDT sensors are difficult and expensive compared to the accelerometer. Also the reason for not using the acceleration signal directly is that firstly it drifts when integrating the signal and the second is the observer concept could be extended more complex systems to estimate the states.

Assume that there are two accelerometers, which are placed on the basement and mass to measure the accelerations of the basement and the mass. The question that arises here is whether an observer is able to estimate the velocity and displacement data of the base and the mass or not. In order to answer this question firstly, the state space representation of the SDOF system is derived as in Equations (9-13) then followed by deciding the observer gain, designing Simulink model of the observer, and lastly comparing the observed data with the actual.

If the estimated values of data are acceptable then the target is to estimate the force produced by the MR damper $f_{m r}$, which is installed between the basement and mass to absorb the vibration by different values of applied current. If the force acting on the MR damper can be 
estimated, then the control algorithm could be developed to control the current applied to the MR damper according to optimal force feedback linearization.

With reference to Figure 1, the observed damper force was obtained using observations of the base $\left(\hat{x}_{b}\right)$ and response motions $\left(\hat{x}_{a}\right)$, along with knowledge of the spring stiffness and payload mass:

$$
f_{m r}=m_{a} \ddot{x}_{a}-k_{a}\left(\hat{x}_{a}-\hat{x}_{b}\right)
$$

This requires estimates of the payload mass acceleration and displacement, as well as the base motion. These were obtained by designing a Luenberger observer [2], using $x_{b}$ as system inputs, $\ddot{x}_{a}$ as available output measurements, and $\hat{x}_{a}$ and $\hat{x}_{b}$ as the states to be identified. The observer error dynamics were placed at $s_{1}=-200$ and $s_{2}=-100$, such that the observer dynamics were considerably faster than the dynamics of the SDOF system $\left(\omega_{n}=24.32 \mathrm{rad} / \mathrm{sec}\right)$.

The full state observer has the form,

$$
\begin{gathered}
\dot{\hat{x}}(t)=\mathrm{A} \hat{x}(t)+\mathrm{B} f_{m r}(t)+\mathrm{E} d(t)+L z . \\
\hat{y}(t)=\mathrm{C} \hat{x}(t)+\mathrm{D} f_{m r}(t)+\mathrm{G} d(t) . \\
z=y-\hat{y} .
\end{gathered}
$$

The dynamics of the state estimation error are then given by

$$
\dot{e}(t)=(\mathrm{A}-\mathrm{LC}) e(t)
$$

Where $\mathrm{L}$ is the observer gain, and

$$
e(t)=x(t)-\hat{x}(t)
$$

If the eigen-values of (A-LC) have negative real parts then $e(t) \rightarrow 0$ as $t \rightarrow \infty$. Hence, $\hat{x}(t) \rightarrow x(t)$ as $t \rightarrow \infty$.

In this study, estimated values of the states are shown to be quite a good match with the actual data after doing several numerical tests.

\section{OPTIMAL FORCE FEEDBACK LINEARIZATION}

The non-linear behaviour of smart fluid dampers makes the objective of achieving a desired force very difficult. Researchers at The University of Sheffield [10] have developed one solution to this problem using feedback linearization, which is briefly summarized below.

The control strategy is shown in block diagram form in Figure 3. Here, feedback control is being used to implement a semi-active force generator. The proposed control system uses measurement of the damper force to linearise the nonlinear damping behaviour. Essentially, the controller gains B and G can be tuned so that the actual force closely matches the set point force. 
In Figure 3, this set-point force is chosen to be proportional to piston velocity by the control gain $\mathrm{D}$, so that the MR damper behaves as a linear viscous device. The values of $\mathrm{B}$ and $\mathrm{G}$ were previously determined through extensive simulation testing on the MR damper, which led to the feedback controller gain $\mathrm{B}=0.6 \mathrm{~N} / \mathrm{N}$ and the feed forward-gain $\mathrm{G}=0.0015 \mathrm{~A} / \mathrm{N}$.

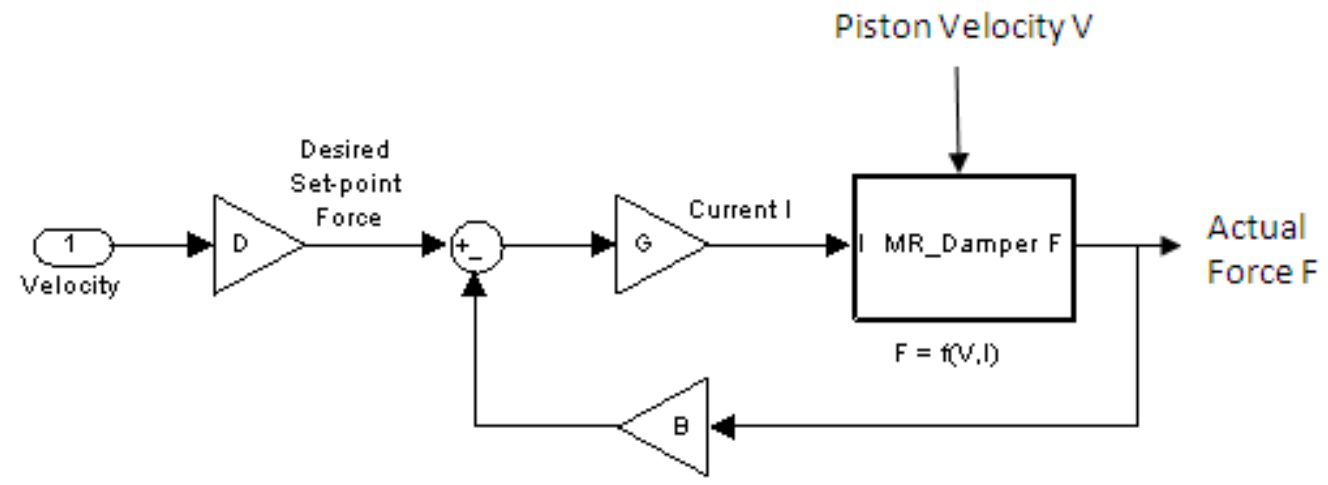

Figure 3: Force-feedback Linearization Control.

In this study, in order improve the performance of the system, the desired set point force is chosen to be the optimal control force. The implementation of the optimal control theory requires the measurement of the system states which are the absolute displacement and velocity of the mass, which are provided by the observer as well.

The approach proposed here is to append a force-feedback loop to induce the MR damper to produce approximately a desired control force $f_{c}$. A linear optimal controller $\mathrm{K}$ is then designed that provides the desired control force $f_{c}$ based on the measured states.

$$
f_{c}=\mathrm{K}\left[\begin{array}{l}
\hat{x}_{a} \\
\hat{\hat{x}}_{a}
\end{array}\right] .
$$

This optimal control force is not possible to be achieved by the MR damper in active region where the energy is injected to the system by the force generator. Due to this passivity limitation of the MR damper, the set point force is set to zero at the active region, by checking the product of the estimated damper force and the estimated piston velocity. This has to be positive to satisfy the passivity theory of Karnopp [4]:

$$
f_{c}=\left\{\begin{array}{cc}
\mathrm{K}\left[\begin{array}{c}
\hat{x}_{a} \\
\dot{\hat{x}}_{a}
\end{array}\right] & f_{c}\left(\dot{\hat{x}}_{a}-\dot{\hat{x}}_{b}\right) \geq 0 \\
0 & \text { otherwise }
\end{array}\right\} .
$$

The optimal gain $\mathrm{K}$ is obtained by the LQR strategies because of their successful application in other engineering structural control applications $[1,13-14,16]$. The matrix $\mathrm{K}$ is the full state feedback gain for deterministic regulator problem given by [14];

$$
\mathrm{K}=\mathrm{B}^{\prime} \mathrm{P} / \mathrm{R} \text {. }
$$


Here $\mathrm{P}$ is the solution of the algebraic Ricatti equation given by

$$
0=\mathrm{PA}+\mathrm{A}^{\prime} \mathrm{P}-\mathrm{PB}^{\prime} \mathrm{BP} / \mathrm{R}+\mathrm{C}^{\prime} \mathrm{Q} \mathrm{C} .
$$

Here $\mathrm{Q}$ and $\mathrm{R}$ weighting matrices minimize the performance index;

$$
J=\int_{0}^{\infty}\left(x^{\prime} \mathrm{Q} x+f^{\prime} \mathrm{R} f\right) d t
$$

After several numerical investigation the best values of the weighting matrices are found as, $\mathrm{Q}=\left[\begin{array}{ll}1 & 0 \\ 0 & 1\end{array}\right]$, and $\mathrm{R}=3.1623 \mathrm{e}-008$.

A block diagram of this semi-active control system is shown in Figure 4. Whole system has four parts as seen in Figure 4: plant, observer, optimal controller, and force-feedback linearization.

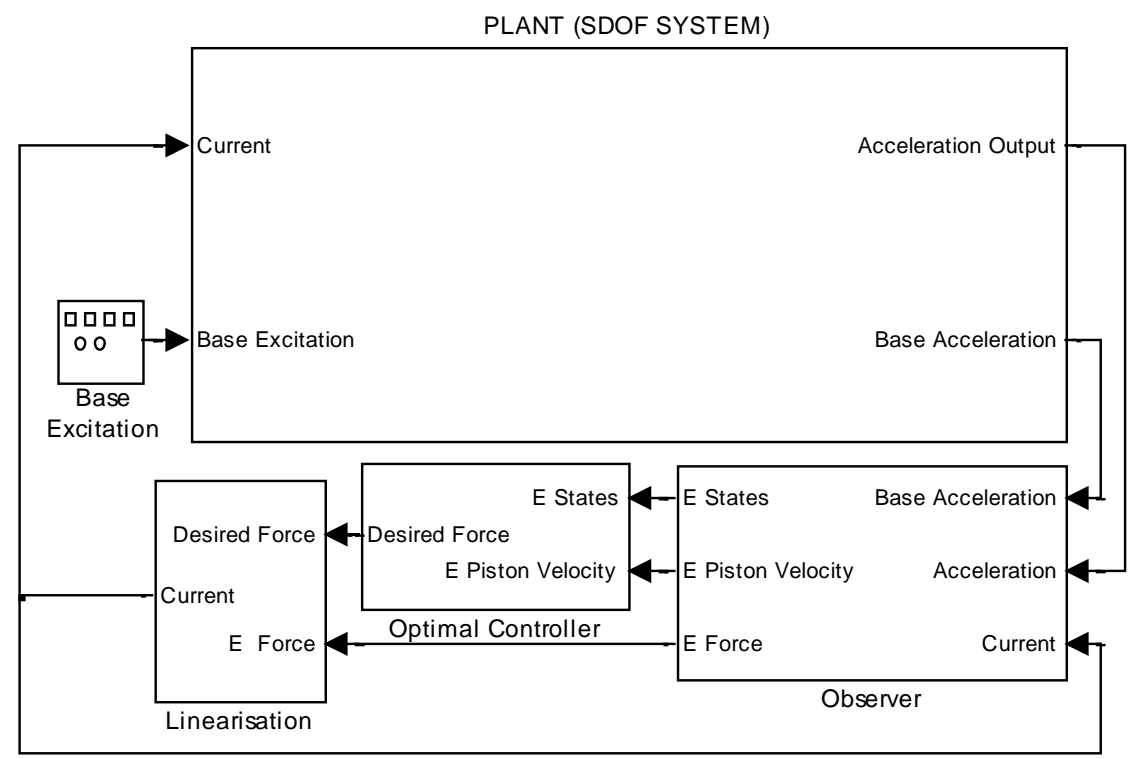

Figure 4: Block Diagram of the Semi-Active Control System. Plant is shown in Figure 1.

\section{NUMERICAL EXAMPLES}

The performance of the optimal force-feedback control algorithm presented in this paper is now evaluated through the numerical simulation. As seen in Figure 1 SDOF mass isolator system is chosen with structural mass $m_{a}=106 \mathrm{~kg}$, and spring stiffness $k_{a}=62 \mathrm{kN} / \mathrm{m}$ also the parameters of the MR damper are $k=13000 \mathrm{kN} / \mathrm{m}$, and $m_{1}=2 \mathrm{~kg}$.

In simulation, the model of the structure is subjected to the sinusoidal base excitation with $0.02 \mathrm{~m}$ amplitude and frequency range of $0.1-6 \mathrm{~Hz}$. In Figure 5 four different displacement transmissibilities of the SDOF system are shown. The square line ( $\square$ ) represents the frequency response of the SDOF system which has a passive damper element with the damping ratio of 
$\zeta=0.39$ instead of the controllable MR damper. The solid line (-) represents the response of the proposed observer based optimal control and dotted line (...) shows the response of the optimal control theory with actual states instead of the estimated. Lastly, the circle line (O) indicates the frequency response of the Sky-Hook control.

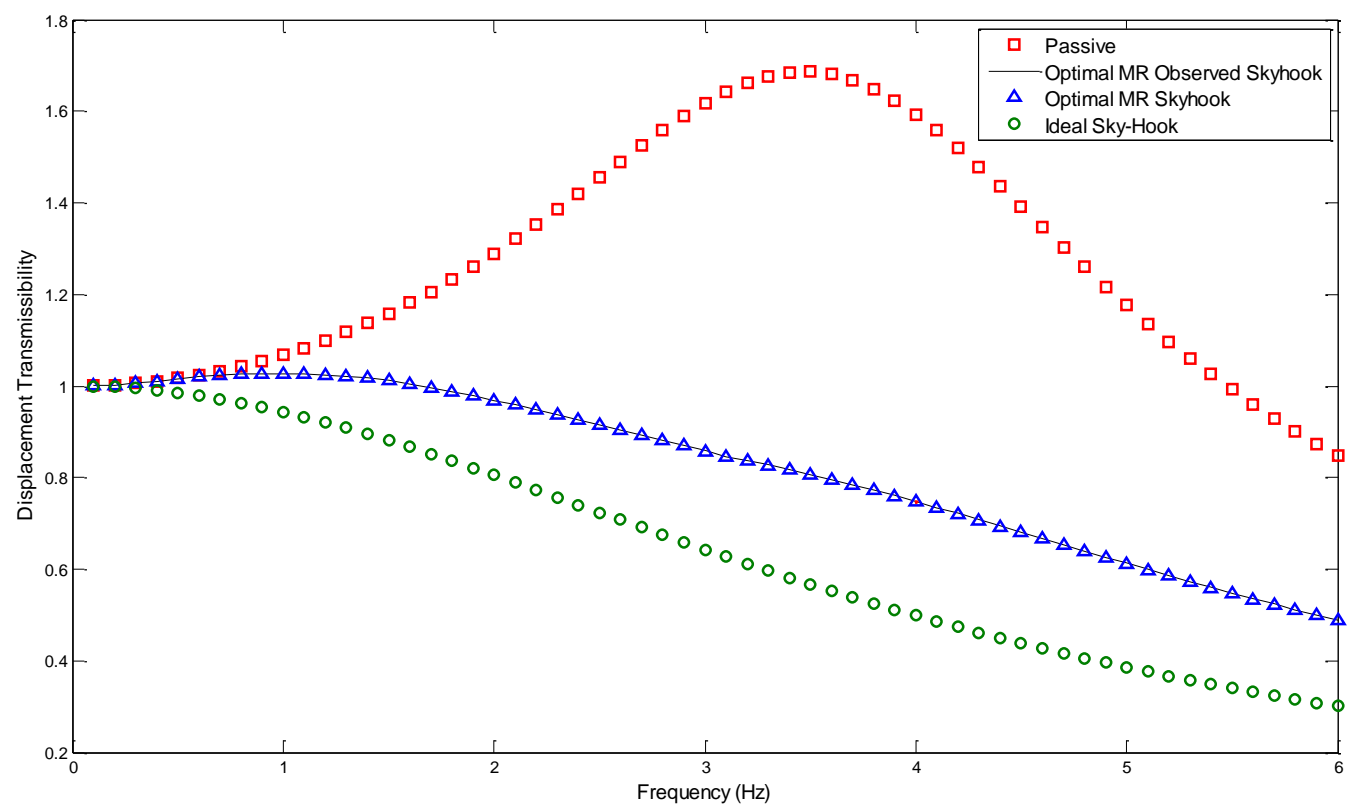

Figure 5: Displacement Transmissibilities of the SDOF system for different control algorithms.

In order to investigate the effect of the weighting matrices, simulations of the proposed control algorithm are run for different $\mathrm{R}$ and $\mathrm{Q}$ values. Figure 6 shows the change of the $\mathrm{R}$ values while keeping the $\mathrm{Q}$ matrices identity. Figure 7 represents the two different performance of the proposed system for two different $\mathrm{Q}$ matrices while $\mathrm{R}$ is equal to $3.1623 e-008$. 


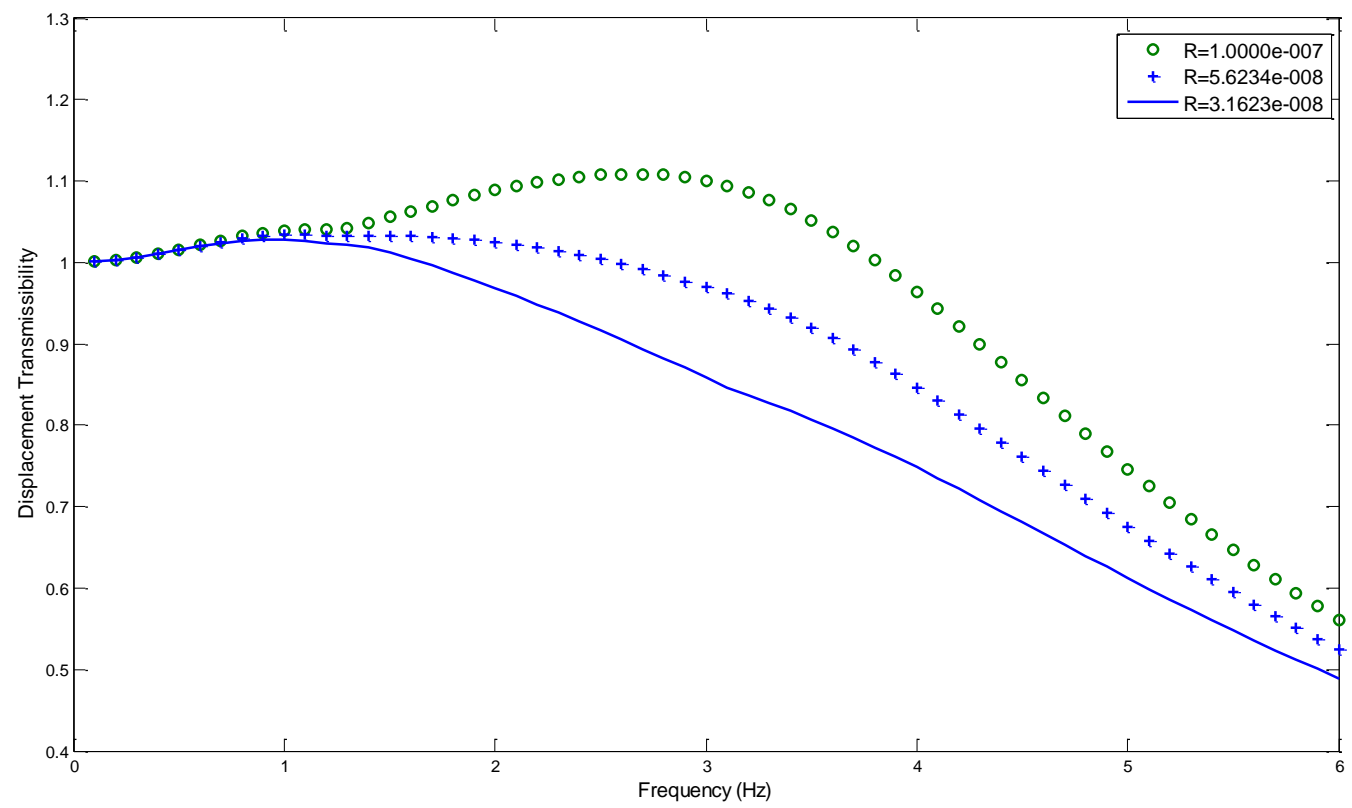

Figure 6: Effect of the different R parameters on the performance of the observer based system, with identity Q matrix.

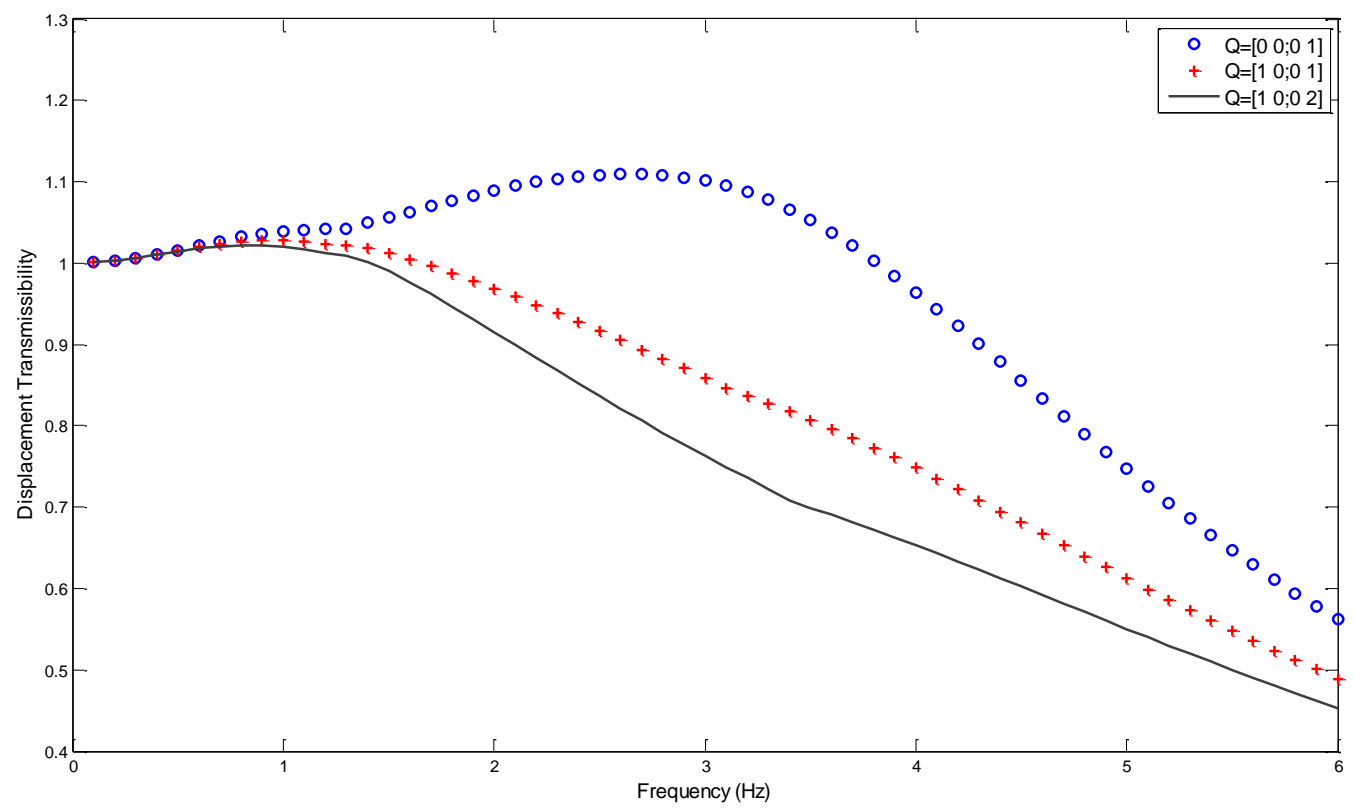

Figure 7: Effect of the different Q parameters on the performance of the observer based system, with $\mathrm{R}=3.1623 e-008$. 


\section{DISCUSSION AND CONCULUSIONS}

The frequency response comparison shown in Figure 5 indicates that the observer is able to estimate the states perfectly: the frequency responses of the observer based optimal forcefeedback control (一) and the optimal force-feedback control based on actual states ( $\cdots$ ) are matching very well. The proposed optimal control algorithm is almost able to push the amplitude of the displacement transmissibility of the system below unit for all frequencies. At the lower frequencies performance of the proposed control is similar to passive control ( $\square$ ) and forcefeedback control $(\boldsymbol{\Delta})$ but for the higher frequencies the performance approaches that of ideal Sky-Hook control (O).

A key issue with the observer-based system is the choice of numerical values for the weighting parameters $\mathrm{Q}$ and $\mathrm{R}$. The positive definite $\mathrm{R}$ matrix accounts for the expenditure of energy of the control signal, and the positive-semidefinite $\mathrm{Q}$ determines the relative importance of the states on control force [6]*. As seen in Figure 6, reducing the value of $\mathrm{R}$ increases the control force and, the proposed algorithm approaches the ideal sky-hook scenario. In Figure 7, for the circle line ( 0 ) Q is chosen as $\left[\begin{array}{llll}0 & 0 & 0 & 1\end{array}\right]$. The second state (velocity) controls the system. For the line (+), $\mathrm{Q}$ is chosen identity matrix so both of the states (displacement and velocity) control the system together, and for the solid line $\mathrm{Q}$ is chosen as $\left[\begin{array}{lll}1 & 0 ; & 0\end{array}\right]$. The velocity has the major effect on the control system. Further work should investigate the implication of these design parameters on practical applications.

In conclusion the observer based optimal force-feedback control algorithm is a promising control strategy for smart fluid dampers. Future work will compare the performance of the proposed control system with the clipped optimal control algorithm developed by Dyke`s et.al. [15]. Furthermore will investigate experimental implementation of proposed control algorithm.

\section{REFERENCES}

[1] B. Spencer Jr, et al., "Frequency domain optimal control strategies for aseismic protection," Journal of Engineering Mechanics, vol. 120, p. 135, 1994.

[2] D. G. Luenberger, "Observing state of linear system," IEEE Transactions on Military Electronics, vol. MIL8, pp. 74-\&, 1964.

[3] D. Karnopp, "Active and semi-active vibration isolation," Journal of Mechanical Design, vol. 117, p. 177, 1995.

[4] D. Karnopp, et al., "Vibration control using semi-active force generators," Journal of Engineering for Industry, vol. 96, pp. 619-626, 1974.

*For this problem $\mathrm{R}$ is scalar. 
[5] J. P. Den Hartog, "Mechanical vibrations,” Dover Pubns, 1985.

[6] K. Ogata, "Modern control engineering," Prentice Hall PTR, 2001.

[7] N. Sims and R. Stanway, "Semi-active vehicle suspension using smart fluid dampers: a modelling and control study," International Journal of Vehicle Design, vol. 33, pp. 76-102, 2003.

[8] N. D. Sims, et al., "A unified modelling and model updating procedure for electrorheological and magnetorheological vibration dampers," Smart Materials \& Structures, vol. 13, pp. 100121, Feb 2004.

[9] N. D. Sims, et al., "Controllable viscous damping: an experimental study of an electrorheological long-stroke damper under proportional feedback control," Smart Materials and Structures, vol. 8, p. 601, 1999.

[10] N. D. Sims, et al., "Smart fluid damping: Shaping the force/velocity response through feedback control," Journal of Intelligent Material Systems and Structures, vol. 11, pp. 945958, Dec 2000.

[11] N. D. Sims, et al., "Vibration control using smart fluids: a state-of-the-art review," The Shock and vibration digest, vol. 31, pp. 195-203, 1999.

[12] R. Sharp and S. Hassan, "The relative performance capabilities of passive, active and semi-active car suspension systems," ARCHIVE: Proceedings of the Institution of Mechanical Engineers, Part D: Transport Engineering 1984-1988 (vols 198-202), vol. 200, pp. 219-228, 1986.

[13] S. Dyke and N. C. f. E. E. Research, "Experimental verification of acceleration feedback control strategies for an active tendon system,": National Center for Earthquake Engineering Research, 1994.

[14] S. Dyke, et al., "Implementation of an active mass driver using acceleration feedback control," Computer-Aided Civil and Infrastructure Engineering, vol. 11, pp. 305-323, 1996.

[15] S. Dyke, et al., "Modeling and control of magneto-rheological dampers for seismic response reduction," Smart Materials and Structures, vol. 5, p. 565, 1996.

[16] S. Dyke, et al., "Role of control-structure interaction in protective system design," Journal of Engineering Mechanics, vol. 121, pp. 322-338, 1995.

[17] S. Timoshenko, "Vibration problems in engineering," 1974. 